Proceedings of the 2019 Winter Simulation Conference

N. Mustafee, K.-H.G. Bae, S. Lazarova-Molnar, M. Rabe, C. Szabo, P. Haas, and Y.-J. Son, eds.

\title{
A PROXIMAL-POINT ALGORITHM WITH VARIABLE SAMPLE-SIZES (PPAWSS) FOR MONOTONE STOCHASTIC VARIATIONAL INEQUALITY PROBLEMS
}

\author{
Afrooz Jalilzadeh \\ Uday V. Shanbhag \\ Department of Industrial and Manufacturing Engineering, \\ Pennsylvania State University \\ University Park, PA 16802, USA.
}

\begin{abstract}
We consider a stochastic variational inequality (SVI) problem with a continuous and monotone mapping over a closed and convex set. In strongly monotone regimes, we present a variable sample-size averaging scheme (VS-Ave) that achieves a linear rate with an optimal oracle complexity. In addition, the iteration complexity is shown to display a muted dependence on the condition number compared with standard variance-reduced projection schemes. To contend with merely monotone maps, we develop amongst the first proximal-point algorithms with variable sample-sizes (PPAWSS), where increasingly accurate solutions of strongly monotone SVIs are obtained via (VS-Ave) at every step. This allows for achieving a sublinear convergence rate that matches that obtained for deterministic monotone VIs. Preliminary numerical evidence suggests that the schemes compares well with competing schemes.
\end{abstract}

\section{INTRODUCTION}

Variational inequality problems have a broad range of applications in engineering, economics, and the applied sciences. Amongst the most common instances of where such problems assume relevance is in the minimization of a differentiable convex function $f$ over a closed and convex set. Recall that $x$ is a minimizer if and only if $(y-x)^{T} \nabla_{x} f(x) \geq 0$ for all $y \in X$, a variational inequality problem succinctly denoted by $\mathrm{VI}\left(X, \nabla_{x} f\right)$. More generally, the variational inequality problem $\mathrm{VI}(X, F)$ requires an $x \in X$ such that

$$
(y-x)^{T} F(x) \geq 0, \quad \forall y \in X .
$$

In addition, such problems find application in modeling saddle-point problems, convex Nash games, traffic equilibrium problems, economic equilibrium problems, amongst others (see Facchinei and Pang (2003)). While deterministic variants (Facchinei and Pang 2003) have seen received significant study over the last several decades, less is known regarding the stochastic variant where the map is replaced by its expectation-valued counterpart. Such a problem, denoted by $\operatorname{SVI}(X, F)$, requires finding an $x \in X$ such that

$$
(y-x)^{T} F(x) \geq 0, \quad \forall y \in X,
$$

where $F(x) \triangleq \mathbb{E}[G(x, \xi)], \xi: \Omega \rightarrow \mathbb{R}^{d}, G: X \times \mathbb{R}^{d} \rightarrow \mathbb{R}^{n}$, and the associated probability space is denoted by $(\Omega, \mathscr{F}, \mathbb{P})$.

Amongst the earliest schemes for resolving $\operatorname{SVI}(X, F)$ via stochastic approximation was presented by Jiang and $\mathrm{Xu}$ (2008) for strongly monotone maps. Regularized variants were developed by Koshal et al. (2013) for merely monotone regimes while Lipschitzian requirements were weakened by combining local smoothing with regularization by Yousefian et al. (2013) and Yousefian et al. (2017). A rate statement of $\mathscr{O}(1 / \sqrt{k})$ for the merely monotone regime (in terms of a suitably defined gap function defined in (3)) was first provided by Juditsky et al. (2011), where $k$ denotes the number of iterations; this appears to have been the best known rate for monotone stochastic variational inequality problems. 
When sampling is "cheap", recent efforts have attempted to reduce the gap between the rates of convergence between deterministic schemes and their stochastic counterparts. In particular, by increasing the sample-size associated with approximating the gradient at a suitable pace, the rate may be improved. In the context of strongly convex stochastic optimization, linear rates of convergence have been proven in both smooth (see Shanbhag and Blanchet (2015) and Jofré and Thompson (2019)), and a subclass of nonsmooth settings (see Jalilzadeh et al. (2018)). Optimal rate statements of $\mathscr{O}\left(1 / k^{2}\right)$ via accelerated variance-reduced schemes in stochastic convex differentiable settings were developed by Ghadimi and Lan (2013) and Jofré and Thompson (2019) matching the best known deterministic rate. Similarly, a rate of $\mathscr{O}(1 / k)$ was provided for nonsmooth but smoothable stochastic convex optimization problems by Jalilzadeh et al. (2018). To the best of our knowledge, the only variance-reduced scheme for monotone stochastic variational inequality problems was presented by Iusem et al. (2017) and is equipped with a rate of $\mathscr{O}(1 / k)$, matching the deterministic rate for monotone variational inequality problems. It is worth emphasizing that the monotone stochastic variational inequality problems can capture stochastic convex optimization problems (with possibly expectation-valued problems), stochastic convex Nash games, and a range of stochastic equilibrium problems. In fact, stochastic convex optimization problems can be solved via acceleration and variance reduction (Jofré and Thompson 2019). However, variational inequality problems are generally not equipped with an appropriate with a natural "objective" function but the gap function (which is generally nonsmooth) serves as a metric for measuring progress of a scheme. The best known rate for monotone deterministic variational inequality problems is $\mathscr{O}(1 / k)$ (in terms of a gap function); recall that a monotone variational inequality problem can be recast as nonsmooth stochastic convex problem (for which the best known rate under smoothability requirements is $\mathscr{O}(1 / k)$ in function values).

(I). Linearly convergent schemes for strongly monotone SVIs. When $F(x)$ is strongly monotone over $X$, i.e. $F(x)-F(y), x-y\rangle \geq \mu\|x-y\|^{2}$ for all $x, y \in X$, the unique solution $x^{*}$ of (2) satisfies the following for all $y \in X$.

$$
\left\langle F(y), x^{*}-y\right\rangle+\frac{1}{2} \mu\left\|y-x^{*}\right\|^{2} \stackrel{[\text { Strong. monot }]}{\leq} \overbrace{\left\langle F\left(x^{*}\right), x^{*}-y\right\rangle}^{\leq 0}-\frac{1}{2} \mu\left\|y-x^{*}\right\|^{2} \stackrel{\left[x^{*} \in \operatorname{soL}(X, F)\right]}{\leq} 0 .
$$

Recall that a gap function associated with (1), denoted by $g: \mathbb{R}^{n} \rightarrow \mathbb{R}$, is a nonnegative function on $X$ and $g\left(x^{*}\right)=0$ for $x^{*} \in \mathbb{R}^{n}$ if and only if $x^{*}$ is a solution of $\operatorname{SVI}$. Therefore, solving $\operatorname{SVI}(X, F)$ is equivalent to minimizing a gap function on $X$. Inspired by Nesterov and Scrimali (2011), we define $g(x)$ as follows.

$$
g(x) \triangleq \sup _{y \in X}\left\{\langle F(y), x-y\rangle+\frac{1}{2} \mu\|y-x\|^{2}\right\} .
$$

Nesterov and Scrimali (2011) proposed a linearly convergent averaging scheme that is reliant on two projections at every step, the first of which is a projection of an averaged vector while the second is the more standard projection. We apply this avenue with two key distinctions: (i) We utilize a noise-corrupted estimate of the expectation-valued map; and (ii) variance reduction is employed to reduce the bias as $k$, the iteration counter. In Section 2, we show that the resulting variable sample-size averaging (VS-Ave) scheme admits a linear rate of convergence when the sample-size is increased at a geometric rate. Importantly, the scheme enjoys a muted dependence on the condition number of the map, in contrast with standard variance-reduced projection schemes and is characterized by an oracle complexity of $\mathscr{O}\left(1 / \varepsilon^{\beta}\right)$ (where $\beta>1$ ) for computing an $\varepsilon$-solution.

(II). Stochastic proximal schemes for monotone SVIs. In section 3, we develop a stochastic generalization of the proximal-point scheme, referred to as a proximal-point algorithm with variable sample-sizes (PPAWSS) for monotone SVIs where the strongly monotone proximal subproblems are solved with increasing accuracy via (VS-Ave). We show that the sequence of iterates converges to the solution at the rate of $\mathscr{O}(1 / k)$, matching the deterministic rate while the iteration complexity in inner projection steps is bounded as $\mathscr{O}\left(\frac{1}{\varepsilon} \log \frac{1}{\varepsilon}\right)$. Preliminary numerics in Section 5 suggest that the scheme compares well with competing techniques. 


\section{LINEARLY CONVERGENT SCHEMES FOR STRONGLY MONOTONE SVI PROBLEMS}

In this section, inspired by Nesterov and Scrimali (2011), we propose a scheme for solving strongly monotone SVIs which admits a linear rate of convergence with a constant of $L / \mu$ in the iteration complexity bound, significantly smaller than $(L / \mu)^{2}$, obtained from standard extensions of projection methods. Our rate statements rely on the following assumptions on $F$.

Assumption 1 Assume that operator $F: X \rightarrow \mathbb{R}^{n}$ is $\mu$-strongly monotone and $L$ - Lipschitz continuous on $X$, i.e. $\|F(x)-F(y)\| \leq L\|x-y\|$ for all $x, y \in X$.

Next, if $\mathscr{F}_{k}$ denotes the information history at epoch $k$, then we have the following requirements on the associated filtrations where $\bar{w}_{k, N_{k}} \triangleq \frac{1}{N_{k}} \sum_{j=1}^{N_{k}}\left(F\left(y_{k}\right)-G\left(y_{k}, \omega_{j, k}\right)\right)$ and $\bar{w}_{k, N_{k}}^{\prime} \triangleq \frac{1}{N_{k}} \sum_{j=1}^{N_{k}}\left(F\left(x_{k}\right)-G\left(x_{k}, \omega_{j, k}\right)\right)$. Assumption 2 There exists $v, \tilde{v}>0$ such that $\mathbb{E}\left[\bar{w}_{k+1, N_{k+1}} \mid \mathscr{F}_{k} \cup \mathscr{F}_{k-1}^{\prime}\right]=0, \mathbb{E}\left[\bar{w}_{k, N_{k}}^{\prime} \mid \mathscr{F}_{k} \cup \mathscr{F}_{k-1}^{\prime}\right]=0$, $\mathbb{E}\left[\left\|\bar{w}_{k+1, N_{k+1}}\right\|^{2} \mid \mathscr{F}_{k} \cup \mathscr{F}_{k-1}^{\prime}\right] \leq \frac{v^{2}}{N_{k+1}}$ and $\mathbb{E}\left[\left\|\bar{w}_{k, N_{k}}^{\prime}\right\|^{2} \mid \mathscr{F}_{k} \cup \mathscr{F}_{k-1}^{\prime}\right] \leq \frac{\tilde{v}^{2}}{N_{k}}$ holds almost surely for all $k$, where $\mathscr{F}_{k} \triangleq \sigma\left\{y_{0}, y_{1}, \ldots, y_{k-1}\right\}, w_{k}^{\prime} \triangleq F\left(x_{k}\right)-G\left(x_{k}, \xi_{k}\right)$ and $\mathscr{F}^{\prime}{ }_{k} \triangleq \sigma\left\{x_{0}, x_{1}, \ldots, x_{k-1}\right\}$.

Throughout the paper, we exploit the following basic lemma stated without proof.

Lemma 1 Given a symmetric positive definite matrix $Q$, we have the following for any $v_{1}, v_{2}, v_{3}$ :

$$
\left(v_{2}-v_{1}\right)^{T} Q\left(v_{3}-v_{1}\right)=\frac{1}{2}\left(\left\|v_{2}-v_{1}\right\|_{Q}^{2}+\left\|v_{3}-v_{1}\right\|_{Q}^{2}-\left\|v_{2}-v_{3}\right\|_{Q}^{2}\right), \text { where }\|v\|_{Q} \triangleq \sqrt{v^{T} Q v} .
$$

Before proceeding, we recall some properties of gap function $g(x)=\sup _{y \in X}\left\{\langle F(y), x-y\rangle+\frac{1}{2} \mu\|y-x\|^{2}\right\}$. Theorem 1 (Nesterov and Scrimali 2011) The gap function $g(x)$ is well defined and $\mu$-strongly convex on $X$. Moreover, $g(x)$ is nonnegative on $X$ and vanishes only at the unique solution of (2).

We present an averaging-based variance-reduced (VS-Ave) scheme (Alg. 1) for strongly monotone SVIs where $K$ and $\Pi_{X}(y)$ denote the total no. of iterations and the projection of $y$ onto $X$, respectively.

Algorithm 1 (VS-Ave $\left(F, y_{0}, \mu, L, K\right)$ ).

(1.0) Given $y_{0} \in X$ and let $\gamma_{0}=1, k=0 ; \Gamma_{0}=1$;

$$
\begin{aligned}
& x_{k}=\Pi_{X}\left[\frac{1}{\sum_{i=0}^{k} \gamma_{i}}\left(\sum_{i=0}^{k} \gamma_{i}\left(y_{i}-\frac{1}{\mu}\left(F\left(y_{i}\right)+\bar{w}_{i, N_{i}}\right)\right)\right)\right] ; \\
& y_{k+1}=\Pi_{X}\left[x_{k}-\left(\frac{1}{L}\right)\left(F\left(x_{k}\right)+\bar{w}_{k, N_{k}}^{\prime}\right)\right] ; \\
& \gamma_{k+1}=\frac{\mu}{\mu+L} \Gamma_{k} ; \Gamma_{k+1}=\Gamma_{k}+\gamma_{k+1} ; \\
& \text { If } k>K, \text { then stop; else } k=k+1 ; \text { return to (1). } \\
& \text { Return } \bar{y}_{k}=\frac{1}{\Gamma_{k}} \sum_{i=0}^{k} \gamma_{i} y_{i} .
\end{aligned}
$$

Remark 1 By definition of the projection in step (1.1) of Algorithm 1, we have that

$$
\left\langle x_{k}-\frac{1}{\sum_{i=1}^{k} \gamma_{i}}\left(\sum_{i=1}^{k} \gamma_{i}\left[y_{i}-\frac{1}{\mu}\left(F\left(y_{i}\right)+\bar{w}_{i, N_{i}}\right)\right]\right), x_{k}-x\right\rangle \leq 0 \quad \text { for all } x \in X .
$$

This can be equivalently represented as $\sum_{i=1}^{k} \gamma_{i}\left\langle F\left(y_{i}\right)+\bar{w}_{i, N_{i}}+\mu\left(x_{k}-y_{i}\right), x_{k}-x\right\rangle \leq 0$ for all $x \in X$, which captures the optimality of $x_{k}$ with respect to $\max _{x \in X}\left\{\sum_{i=0}^{k} \gamma_{i}\left[\left\langle F\left(y_{i}\right)+\bar{w}_{i, N_{i}}, y_{i}-x\right\rangle-\frac{1}{2} \mu\left\|x-y_{i}\right\|^{2}\right]\right\}$.

In the following lemma, we provide an upper bound for the gap function.

Lemma 2 (Nesterov and Scrimali 2011) Consider a sequence of positive weights $\left\{\gamma_{i}\right\}_{i=0}^{K}$ and points $\left\{y_{i}\right\}_{i=0}^{K} \subset$ $\mathbb{R}^{n}$. Let $\Gamma_{K}=\sum_{i=0}^{K} \gamma_{i}, \bar{y}_{K}=\frac{1}{\Gamma_{k}} \sum_{i=0}^{K} \gamma_{i} y_{i}$ and $\tilde{\mathscr{A}}_{K}=\max _{x \in X}\left\{\sum_{i=0}^{K} \gamma_{i}\left[\left\langle F\left(y_{i}\right), y_{i}-x\right\rangle-\frac{1}{2} \mu\left\|x-y_{i}\right\|^{2}\right]\right\}$. Then, $g\left(\bar{y}_{K}\right) \leq \frac{1}{\Gamma_{K}} \tilde{A}_{K}$.

Next, we derive a bound on the conditional expectation on $\mathscr{A}_{k+1}$. 
Lemma 3 Consider the sequences generated by Algorithm 1. Suppose Assumptions 1 and 2 hold, and $\mathscr{A}_{k} \triangleq \max _{x \in X}\left\{\sum_{i=0}^{k} \gamma_{i}\left[\left\langle F\left(y_{i}\right)+\bar{w}_{i, N_{i}}, y_{i}-x\right\rangle-\frac{1}{2} \mu\left\|x-y_{i}\right\|^{2}\right]\right\}$. Then the following holds for all $k$.

$$
\mathbb{E}\left[\mathscr{A}_{k+1} \mid \mathscr{F}_{k} \cup \mathscr{F}_{k-1}^{\prime}\right] \leq \mathscr{A}_{k}+\gamma_{k+1}\left(\frac{1}{c}+\frac{1}{\mu}\right)\left(\frac{v^{2}}{N_{k+1}}+\frac{(\tilde{v})^{2}}{N_{k}}\right) \text {, where } c \triangleq \frac{L \mu}{L+\mu} .
$$

Proof. From the definition of $\mathscr{A}_{k+1}$ we have that:

$$
\begin{aligned}
\mathscr{A}_{k+1} & =\max _{x \in X}\{\sum_{i=0}^{k} \gamma_{i} \underbrace{\left[\left\langle F\left(y_{i}\right)+\bar{w}_{i, N_{i}}, y_{i}-x\right\rangle-\frac{1}{2} \mu\left\|x-y_{i}\right\|^{2}\right]}_{\text {Term (A) }} \\
& +\gamma_{k+1} \underbrace{\left[\left\langle F\left(y_{k+1}\right)+\bar{w}_{k+1, N_{k+1}}, y_{k+1}-x\right\rangle-\frac{1}{2} \mu\left\|x-y_{k+1}\right\|^{2}\right]}_{\text {Term (B) }}\} .
\end{aligned}
$$

One can easily check that term $(A)$ in (4) is $\mu$-strongly concave in $x$. Therefore, we obtain the following:

$$
\begin{aligned}
& \sum_{i=0}^{k} \gamma_{i}\left[\left\langle F\left(y_{i}\right)+\bar{w}_{i, N_{i}}, y_{i}-x\right\rangle-\frac{\mu}{2}\left\|x-y_{i}\right\|^{2}\right]=\sum_{i=0}^{k} \gamma_{i}\left[\left\langle F\left(y_{i}\right)+\bar{w}_{i, N_{i}}, y_{i}-x_{k}\right\rangle-\frac{\mu}{2}\left\|x_{k}-y_{i}\right\|^{2}\right] \\
& +\sum_{i=0}^{k} \gamma_{i}\left[\left\langle F\left(y_{i}\right)+\bar{w}_{i, N_{i}}+\mu\left(x_{k}-y_{i}\right), x_{k}-x\right\rangle-\frac{\mu}{2}\left\|x-x_{k}\right\|^{2}\right] \leq \mathscr{A}_{k}-\frac{\mu \Gamma_{k}}{2}\left\|x-x_{k}\right\|^{2}, \quad \forall x \in X,
\end{aligned}
$$

where in the last inequality, we used $\sum_{i=1}^{k} \gamma_{i}\left\langle F\left(y_{i}\right)+\bar{w}_{i, N_{i}}+\mu\left(x_{k}-y_{i}\right), x_{k}-x\right\rangle \leq 0$ for all $x \in X$ from Remark 1 and $\Gamma_{k}=\sum_{i=0}^{k} \gamma_{i}$. From the definition of $y_{k}$ in Algorithm 1,

$$
\left\langle F\left(x_{k}\right)+\bar{w}_{k, N_{k}}^{\prime}+L\left(y_{k+1}-x_{k}\right), y_{k+1}-x\right\rangle \leq 0 \text { for all } x \in X .
$$

Consequently, Term (B) can be bounded as follows by using (6) and the Cauchy-Schwarz inequality:

$$
\begin{aligned}
& \left\langle F\left(y_{k+1}\right)+\bar{w}_{k+1, N_{k+1}}, y_{k+1}-x\right\rangle-\frac{\mu}{2}\left\|x-y_{k+1}\right\|^{2} \\
& =\left\langle F\left(y_{k+1}\right)-F\left(x_{k}\right), y_{k+1}-x\right\rangle-\frac{\mu}{2}\left\|x-y_{k+1}\right\|^{2}+\left\langle F\left(x_{k}\right)+\bar{w}_{k, N_{k}}^{\prime}, y_{k+1}-x\right\rangle+\left\langle\bar{w}_{k+1, N_{k+1}}-\bar{w}_{k, N_{k}}^{\prime}, y_{k+1}-x\right\rangle \\
& \stackrel{(6)}{\leq}\left\|F\left(y_{k+1}\right)-F\left(x_{k}\right)\right\|\left\|y_{k+1}-x\right\|-\frac{\mu}{2}\left\|x-y_{k+1}\right\|^{2}+L\left\langle\left(y_{k+1}-x_{k}\right), x-y_{k+1}\right\rangle \\
& +\left\langle\bar{w}_{k+1, N_{k+1}}-\bar{w}_{k, N_{k}}^{\prime}, y_{k+1}-x\right\rangle .
\end{aligned}
$$

Applying Lemma 1 with $Q=I$, we have that the penultimate term can be expressed as follows.

$$
2\left\langle\left(y_{k+1}-x_{k}, x-y_{k+1}\right)\right\rangle=\left\|x-x_{k}\right\|^{2}-\left\|y_{k+1}-x_{k}\right\|^{2}-\left\|x-y_{k+1}\right\|^{2} .
$$

Hence, using (8) and the fact that $a^{T} b \leq \frac{1}{2(L+\mu)}\|a\|^{2}+\frac{L+\mu}{2}\|b\|^{2}$, inequality (7) can be written as follows:

$$
\begin{aligned}
& \left\langle F\left(y_{k+1}\right)+\bar{w}_{k+1, N_{k+1}}, y_{k+1}-x\right\rangle-\frac{\mu}{2}\left\|x-y_{k+1}\right\|^{2} \\
& \leq \frac{1}{2(L+\mu)}\left\|F\left(y_{k+1}\right)-F\left(x_{k}\right)\right\|^{2}+\frac{(L+\mu)}{2}\left\|y_{k+1}-x\right\|^{2}-\frac{\mu}{2}\left\|x-y_{k+1}\right\|^{2} \\
& +\frac{L}{2}\left\|x-x_{k}\right\|^{2}-\frac{L}{2}\left\|y_{k+1}-x_{k}\right\|^{2}-\frac{L}{2}\left\|x-y_{k+1}\right\|^{2}+\left\langle\bar{w}_{k+1, N_{k+1}}-\bar{w}_{k, N_{k}}^{\prime}, y_{k+1}-x\right\rangle \\
& =\frac{1}{2(L+\mu)}\left\|F\left(y_{k+1}\right)-F\left(x_{k}\right)\right\|^{2}+\frac{L}{2}\left\|x-x_{k}\right\|^{2}-\frac{L}{2}\left\|y_{k+1}-x_{k}\right\|^{2}+\left\langle\bar{w}_{k+1, N_{k+1}}-\bar{w}_{k, N_{k}}^{\prime}, y_{k+1}-x\right\rangle .
\end{aligned}
$$


where the last term in (9) can be written as

$$
\begin{aligned}
\left\langle\bar{w}_{k+1, N_{k+1}}-\bar{w}_{k, N_{k}}^{\prime}, y_{k+1}-x_{k}+x_{k}-x\right\rangle & \leq \frac{1}{c}\left(\left\|\bar{w}_{k+1, N_{k+1}}\right\|^{2}+\left\|\bar{w}_{k, N_{k}}^{\prime}\right\|^{2}\right)+\frac{c}{2}\left\|y_{k+1}-x_{k}\right\|^{2} \\
& +\frac{1}{\mu}\left(\left\|\bar{w}_{k+1, N_{k+1}}\right\|^{2}+\left\|\bar{w}_{k, N_{k}}^{\prime}\right\|^{2}\right)+\frac{\mu}{2}\left\|x_{k}-x\right\|^{2},
\end{aligned}
$$

where $c=\frac{L \mu}{L+\mu}<L$. It follows that by utilizing (10) in (9) and then substituting the result and (5) in (4) and by recalling that $\gamma_{k+1}=(\mu /(L+\mu)) \Gamma_{k}$, we get the following:

$$
\begin{aligned}
\mathscr{A}_{k+1} & \leq \mathscr{A}_{k}-\frac{\mu \Gamma_{k}}{2}\left\|x-x_{k}\right\|^{2}+\gamma_{k+1}\left[\frac{1}{2(L+\mu)}\left\|F\left(y_{k+1}\right)-F\left(x_{k}\right)\right\|^{2}+\frac{L+\mu}{2}\left\|x-x_{k}\right\|^{2}-\frac{L-c}{2}\left\|y_{k+1}-x_{k}\right\|^{2}\right. \\
& \left.+\left(\frac{1}{c}+\frac{1}{\mu}\right)\left(\left\|\bar{w}_{k+1, N_{k+1}}\right\|^{2}+\left\|\bar{w}_{k, N_{k}}^{\prime}\right\|^{2}\right)\right] \\
& \leq \mathscr{A}_{k}+\frac{\gamma_{k+1}}{2}\left[\frac{1}{L+\mu}\left\|F\left(y_{k+1}\right)-F\left(x_{k}\right)\right\|^{2}-(L-c)\left\|y_{k+1}-x_{k}\right\|^{2}\right] \\
& +\gamma_{k+1}\left(\frac{1}{c}+\frac{1}{\mu}\right)\left(\left\|\bar{w}_{k+1, N_{k+1}}\right\|^{2}+\left\|\bar{w}_{k, N_{k}}^{\prime}\right\|^{2}\right) .
\end{aligned}
$$

Now using Assumption 1, we obtain the following.

$$
\frac{1}{L+\mu}\left\|F\left(y_{k+1}\right)-F\left(x_{k}\right)\right\|^{2}-\left\|y_{k+1}-x_{k}\right\|_{L I-c I}^{2} \leq\left\|y_{k+1}-x_{k}\right\|_{\left(\frac{L^{2}}{L+\mu}-L+c\right) I}^{2}=0,
$$

where the last equality follows from $\frac{L^{2}}{\mu+L}+c=L$, a consequence of the definition of $c$. Using (12) and (10) within (11) and taking conditional expectations with respect to $\mathscr{F}_{k} \cup \mathscr{F}_{k-1}^{\prime}$, we obtain

$$
\mathbb{E}\left[\mathscr{A}_{k+1} \mid \mathscr{F}_{k} \cup \mathscr{F}_{k-1}^{\prime}\right] \leq \mathscr{A}_{k}+\gamma_{k+1}\left(\frac{1}{c}+\frac{1}{\mu}\right) \mathbb{E}\left[\left\|\bar{w}_{k+1, N_{k+1}}\right\|^{2}+\left\|\bar{w}_{k, N_{k}}^{\prime}\right\|^{2} \mid \mathscr{F}_{k} \cup \mathscr{F}_{k-1}^{\prime}\right],
$$

which leads to the desired result by using Assumption 2.

We now derive rate and complexity statements for Algorithm 1.

Theorem 2 (Rate and Complexity Statement for (VS-Ave)) Suppose Assumptions 1 and 2 hold. Suppose $\mathscr{A}_{k}$ is as defined in Lemma 3 and $x^{*}$ denotes the solution of (2). Consider the iterates generated by Algorithm 1 and let $N_{k} \triangleq\left\lfloor\rho^{-k}\right\rfloor$ such that $\rho<1-\frac{1}{\kappa+2}$.

(i) If $\kappa \triangleq \frac{L}{\mu}$ and $c \triangleq \frac{L \mu}{L+\mu}$, the following holds for all $K$.

$$
\frac{\mu \mathbb{E}\left[\left\|\bar{y}_{K}-x^{*}\right\|^{2}\right]}{2} \leq\left(g\left(y_{0}\right) \kappa^{2}+2 \kappa\left(v^{2}+\tilde{v}^{2}\right)\left(\frac{1}{c}+\frac{1}{\mu}\right)\left(\frac{\kappa+1}{(\kappa+2)(1-\rho)-1}\right)\right)\left(1-\frac{1}{\kappa+2}\right)^{K}=C q^{K} .
$$

(ii) Suppose $\bar{y}_{K}$ is an $\varepsilon$-solution such that $\mathbb{E}\left[\left\|\bar{y}_{K}-x^{*}\right\|^{2}\right] \leq \varepsilon$. If $\bar{C}=2 C / \mu$, then Algorithm 1 requires $\mathscr{O}(\kappa \log (\bar{C} / \varepsilon))$ steps and $\mathscr{O}(1 / \varepsilon)^{\beta}$ evaluations where $\rho=\bar{q}^{\beta}$ and $\beta>1$.

Proof. (i) From the strong convexity of $g$, we have that $\frac{\mu}{2}\left\|\bar{y}_{k}-x^{*}\right\|^{2} \leq g(\bar{y})$. Note that from Lemma 3, the following holds for all $k$ by taking unconditional expectations.

$$
\begin{aligned}
& \mathbb{E}\left[\mathscr{A}_{k+1} \mid \mathscr{F}_{k} \cup \mathscr{F}_{k-1}^{\prime}\right] \leq \mathscr{A}_{k}+\gamma_{k+1}\left(\frac{1}{c}+\frac{1}{\mu}\right)\left(\frac{v^{2}}{N_{k+1}}+\frac{\tilde{v}^{2}}{N_{k}}\right) \Longrightarrow \mathbb{E}\left[\mathscr{A}_{k+1}\right] \leq \mathbb{E}\left[\mathscr{A}_{k}\right]+\gamma_{k+1}\left(\frac{1}{c}+\frac{1}{\mu}\right)\left(\frac{v^{2}}{N_{k+1}}+\frac{\tilde{v}^{2}}{N_{k}}\right) \\
& \Longrightarrow \mathbb{E}\left[\mathscr{A}_{K}\right] \leq \mathscr{A}_{0}+\sum_{k=0}^{K-1} \gamma_{k+1}\left(\frac{1}{c}+\frac{1}{\mu}\right)\left(\frac{v^{2}}{N_{k+1}}+\frac{\tilde{v}^{2}}{N_{k}}\right)
\end{aligned}
$$




\section{Jalilzadeh and Shanbhag}

Since $\Gamma_{0}=\gamma_{0}=1$, we have that $\Gamma_{k+1}=\Gamma_{k}+\gamma_{k+1}=\Gamma_{k}+\frac{\mu}{\mu+L} \Gamma_{k}=\left(1+\frac{1}{1+\kappa}\right) \Gamma_{k}$. Therefore, using Lemma 2 and the fact that $\max \mathbb{E}[.] \leq \mathbb{E}[\max ()$.$] , the following holds for \bar{y}_{k}=\frac{1}{\Gamma_{k}} \sum_{i=0}^{k} \gamma_{i} y_{i}$.

$$
\mathbb{E}\left[g\left(\bar{y}_{K}\right)\right] \leq \frac{\mathbb{E}\left[\tilde{\mathscr{A}}_{K}\right]}{\Gamma_{K}} \leq \frac{\mathbb{E}\left[\mathscr{A}_{K}\right]}{\Gamma_{K}} \stackrel{(13)}{\leq}\left(\mathscr{A}_{0}+\sum_{k=0}^{K-1} \gamma_{k+1}\left(\frac{1}{c}+\frac{1}{\mu}\right)\left(v^{2} / N_{k+1}+\tilde{v}^{2} / N_{k}\right)\right)\left(1-\frac{1}{\kappa+2}\right)^{K}
$$

where $\frac{1}{\Gamma_{K}}=\left(\frac{\kappa+1}{\kappa+2}\right)^{K}=\left(1-\frac{1}{\kappa+2}\right)^{K}$. Using the definition of $\mathscr{A}_{0}$, we obtain:

$$
\begin{aligned}
\mathscr{A}_{0} & =\max _{x \in X}\left\{\left\langle F\left(y_{0}\right)+F\left(x^{*}\right)-F\left(x^{*}\right), y_{0}-x\right\rangle-\frac{1}{2} \mu\left\|x-y_{0}\right\|^{2}\right\} \\
& =\max _{x \in x} \underbrace{\left\langle F\left(x^{*}\right), x^{*}-x\right\rangle}_{\leq 0 \text { since } x^{*} \in \operatorname{soL}(X, F)}+\left\langle F\left(x^{*}\right), y_{0}-x^{*}\right\rangle+\max _{x \in X}\left\{\left\langle F\left(y_{0}\right)-F\left(x^{*}\right), y_{0}-x\right\rangle-\frac{1}{2} \mu\left\|x-y_{0}\right\|^{2}\right\} \\
& \left.\leq F\left(x^{*}\right), y_{0}-x^{*}\right\rangle+\underbrace{\max _{x \in X}\left\{\left\langle F\left(y_{0}\right)-F\left(x^{*}\right), y_{0}-x\right\rangle-\frac{1}{2} \mu\left\|x-y_{0}\right\|^{2}\right\}}_{\text {Term (C) }} .
\end{aligned}
$$

Since $a^{T} b \leq \frac{1}{2 \mu}\|a\|^{2}+\frac{\mu}{2}\|b\|^{2}$ for $a, b \in \mathbb{R}^{n}$, Term (C) $\leq \max _{x \in X}\left\{\frac{1}{2 \mu}\left\|F\left(y_{0}\right)-F\left(x^{*}\right)\right\|^{2}+\frac{\mu}{2}\left\|y_{0}-x\right\|^{2}-\right.$ $\left.\frac{\mu}{2}\left\|x-y_{0}\right\|^{2}\right\}=\frac{1}{2 \mu}\left\|F\left(y_{0}\right)-F\left(x^{*}\right)\right\|^{2}$. By Lipschitz continuity of $F$ and the definition of $g$, we have that

$$
\begin{aligned}
\mathscr{A}_{0} & \leq\left\langle F\left(x^{*}\right), y_{0}-x^{*}\right\rangle+\frac{L^{2}}{2 \mu}\left\|y_{0}-x^{*}\right\|^{2} \leq g\left(y_{0}\right)-\frac{\mu}{2}\left\|x^{*}-y_{0}\right\|^{2}+\frac{L^{2}}{2 \mu}\left\|y_{0}-x^{*}\right\|^{2} \\
& =g\left(y_{0}\right)+\left\|y_{0}-x^{*}\right\|^{2} \frac{\mu}{2}\left(\frac{L^{2}}{\mu^{2}}-1\right) \leq g\left(y_{0}\right)+g\left(y_{0}\right)\left(\frac{L^{2}}{\mu^{2}}-1\right)=\frac{L^{2}}{\mu^{2}} g\left(y_{0}\right),
\end{aligned}
$$

where the penultimate inequality follows from the strong convexity of $g\left(y_{0}\right)$ and $g\left(x^{*}\right)=0$. Furthermore, using the definition of $\gamma_{k}$, we have the following bounds.

$$
\begin{aligned}
\sum_{k=0}^{K-1} \gamma_{k+1} & \left(\frac{1}{c}+\frac{1}{\mu}\right)\left(\frac{v^{2}}{N_{k+1}}+\frac{\tilde{v}^{2}}{N_{k}}\right)=\kappa\left(\frac{1}{c}+\frac{1}{\mu}\right) \sum_{k=0}^{K-1}\left(1+\frac{1}{\kappa+1}\right)^{k}\left(\frac{v^{2}}{N_{k+1}}+\frac{\tilde{v}^{2}}{N_{k}}\right) \\
\leq & \kappa\left(\frac{1}{c}+\frac{1}{\mu}\right) \sum_{k=0}^{K-1}\left(1+\frac{1}{\kappa+1}\right)^{k}\left(\frac{v^{2}+\tilde{v}^{2}}{N_{k}}\right) \\
\leq & 2 \kappa\left(v^{2}+\tilde{v}^{2}\right)\left(\frac{1}{c}+\frac{1}{\mu}\right) \sum_{k=0}^{K-1} \frac{\rho^{k}}{\left(1-\frac{1}{\kappa+2}\right)^{k}} \leq 2 \kappa\left(v^{2}+\tilde{v}^{2}\right)\left(\frac{1}{c}+\frac{1}{\mu}\right) \frac{1}{1-\frac{\rho}{1-\frac{1}{\kappa+2}}} \\
= & 2 \kappa\left(v^{2}+\tilde{v}^{2}\right)\left(\frac{1}{c}+\frac{1}{\mu}\right)\left(\frac{\kappa+1}{(\kappa+2)(1-\rho)-1}\right) .
\end{aligned}
$$

By substituting (15) and (16) in (14) and by recalling that $\frac{\mu}{2}\left\|\bar{y}_{K}-x^{*}\right\|^{2} \leq g\left(\bar{y}_{K}\right)$, the result follows.

(ii) To obtain $\varepsilon$-solution we need $\bar{C} q^{K} \leq \varepsilon$, where $\bar{C}=2 C / \mu$. Therefore, we need at least $K \geq \log _{1 / q} \bar{C} / \varepsilon=$ $\frac{\log \tilde{C} / \varepsilon}{\log 1 / q}$ steps. From the definition of $q$, we have that $\log (1 / q)=\log \left(1+\frac{1}{\kappa+1}\right) \geq \frac{1}{2 \kappa+2}$ by recalling that $\log (1+x) \geq \frac{x}{2}$ for $x \in[0,1]$. Consequently, the iteration complexity is $\mathscr{O}(\kappa \log (\bar{C} / \varepsilon))$ where $\bar{C} \leq \mathscr{O}\left(\kappa^{2}\left(v^{2}+\right.\right.$ $\left.\tilde{v}^{2}\right)$ ). Similarly, the oracle complexity can be computed as follows for $\rho=q^{\beta}$ and $\beta>1$ :

$$
\sum_{k=0}^{\log _{1 / q} \bar{C} / \varepsilon+1}\left\lfloor\rho^{-k}\right\rfloor=\frac{\rho^{-\log _{1 / q} \bar{C} / \varepsilon-2}-1}{1 / \rho-1} \leq \frac{\rho^{-\log _{1 / q} \bar{C} / \varepsilon-1}}{1-\rho}=\frac{\bar{C}^{\beta}}{\rho-\rho^{2}}(1 / \varepsilon)^{\beta} .
$$


Remark 2 Standard variance-reduced projection schemes for (SVIs) are characterized by an iteration complexity bound of $\mathscr{O}\left(\kappa^{2} \log \left(\frac{1}{\varepsilon}\right)\right)$ while Algorithm 2 has a bound given by $\mathscr{O}\left(\kappa \log \left(\frac{1}{\varepsilon}\right)\right)$.

\section{PROXIMAL SCHEMES FOR MONOTONE STOCHASTIC PROBLEMS}

Monotone stochastic variational inequality problems have been examined extensively in the literature. In monotone regimes, the best known result via variance reduction was provided by Iusem et al. (2017) where it a suitably defined residual function provably diminishes at $\mathscr{O}(1 / k)$ when a stochastic extragradient scheme is employed together with a variance reduction framework. We develop a new avenue for resolving SVIs through a stochastic proximal point framework. We begin by summarizing proximal point schemes for generalized equations in Section 3.1 and then extend and analyze a stochastic generalization in Section 3.2.

\subsection{A Review of Proximal Point Schemes}

Consider the solution of a generalized equation $0 \in T(u)$, where $T: \mathbb{R}^{n} \rightarrow \mathbb{R}^{n}$ is a set-valued maximal monotone operator. A map $T$ is said to be $\mu$-strongly monotone if there exists a $\mu>0$ such that $(v-u)^{T}(y-x) \geq \mu\|y-x\|^{2}, \forall x, y$, where $v \in T(y)$ and $u \in T(x)$. Furthermore, if $\mu=0$, this reduces to mere monotonicity. Recall that the graph of a monotone map is defined as $\operatorname{gph}(T) \triangleq\{(x, y) \mid y \in T(x)\}$. Then $T$ is a maximal monotone operator if no monotone map $\Psi$ exists such that $\operatorname{gph}(T) \subset \operatorname{gph}(\Psi)$. For instance, the subdifferential map $\partial f$ of a closed, convex, and proper function is a maximal monotone map. The classical proximal point algorithm can be traced back to the seminal paper by Rockafellar (1976); given a vector $u_{0} \in \mathbb{R}^{n}$, (17) generates a sequence $\left\{u_{k}\right\}$ defined as follows:

$$
0 \in T\left(u_{k+1}\right)+\frac{1}{\lambda}\left(u_{k+1}-u_{k}\right) .
$$

Consequently, the resolvent operator of a monotone set-valued operator, denoted by $J_{\lambda}^{T}(u)$, is defined as $J_{\lambda}^{T} \triangleq(I+\lambda T)^{-1}$. Then we may state the update rule (17) as an update using the resolvent operator, i.e.

$$
\left[0=T\left(u_{k+1}\right)+\frac{1}{\lambda}\left(u_{k+1}-u_{k}\right)\right] \Longleftrightarrow\left[u_{k+1}=J_{\lambda}^{T}\left(u_{k}\right)\right] .
$$

Consider the generalized proximal point method, rooted in the seminal work by Rockafellar (1976), which employs the following update rule (see Corman and Yuan (2014)):

$$
u_{k+1} \triangleq \eta J_{\lambda}^{T}\left(u_{k}\right)+(1-\eta) u_{k}
$$

which reduces to (17) when $\eta=1$. We now define the Yosida approximation operator $T_{\lambda}$ as $T_{\lambda} \triangleq \frac{\left(I-J_{\lambda}^{T}\right)}{\lambda}$. It has been shown that if $T$ is a monotone set-valued map and $J_{\lambda}^{T}$ denotes its resolvent, then $T_{\lambda}$ is $\lambda$ firmly non-expansive and $\frac{1}{\lambda}$-Lipschitz continuous. Moreover, we have that from Corman and Yuan (2014), Prop. 2.4) that for all $\lambda>0,0 \in T(u) \Leftrightarrow T_{\lambda}(u)=0$. In other words, computing a zero of a generalized equation is equivalent to computing a root of a suitably defined nonlinear equation. By definition of $T_{\lambda}(x)$, we note that (18) can be recast as follows:

$$
u_{k+1}=\eta J_{\lambda}^{T}\left(u_{k}\right)+(1-\eta) u_{k}=u_{k}-\eta\left(u_{k}-J_{\lambda}^{T}\left(u_{k}\right)\right)=u_{k}-\eta \lambda T_{\lambda}\left(u_{k}\right) .
$$

In other words, the update is seen to be similar to a subgradient scheme, where the subgradient of $f$ is replaced by the $T_{\lambda}\left(u_{k}\right)$ while the steplength is given by $\eta \lambda$. We now reproduce the main rate statements available for (18) in both monotone and strongly monotone regimes (see (Corman and Yuan 2014)).

$$
\begin{array}{llrl}
\left\|T_{\lambda}\left(u_{k}\right)\right\|^{2} \leq \mathscr{O}\left(\frac{1}{k+1}\right), & k \geq 0 & \text { ((Monotone), } \gamma \in(0,2)) \\
\left\|u_{k}-u^{*}\right\|^{2} \leq \rho^{k}\left\|u_{0}-u^{*}\right\|^{2}, & k \geq 0, \quad \rho \triangleq\left|1-\frac{\gamma \lambda \alpha}{1+\lambda \alpha}\right| . & \text { (St. Monotone), } \gamma \in(0,2))
\end{array}
$$




\subsection{A Stochastic Proximal Point Framework}

Faced with an expectation-valued map $T(u) \triangleq \mathbb{E}[T(u, \omega)]$, a direct application of (17) is impossible since it requires exactly evaluating the resolvent $J_{\lambda}^{T}$ of an expectation-valued map. Consequently, contending with such problems requires inexactly evaluating the resolvent $J_{\lambda}^{T}$; in effect, we compute a noise-afflicted variant of $T_{\lambda}\left(u_{k}\right)$, given by $T_{\lambda}\left(u_{k}\right)+w_{k}$, where $\mathbb{E}\left[\left\|w_{k}\right\|^{2}\right] \leq \frac{v^{2}}{N_{k}}$, utilizing update rule (19).

$$
u_{k+1}=u_{k}-\eta \lambda\left(T_{\lambda}\left(u_{k}\right)+w_{k}\right)
$$

Our goal lies in inexactly computing $J_{\lambda}^{T}(u)$ when $T(u)$ corresponds to the generalized equation reformulation of a variational inequality problem. Specifically, $x$ is a solution to (1) if and only if

$$
0 \in T(x) \triangleq F(x)+\mathscr{N}_{X}(x)
$$

where $\mathscr{N}_{X}(x)$ refers to the normal cone of $X$ at $x$. is defined as $T(u) \triangleq F(u)+\mathscr{N}_{X}(u)$, where $\mathscr{N}_{X}(u)$ is normal cone of $X$. Recall that when $F$ is a single-valued monotone map, $F(x)+\mathscr{N}_{X}(x)$ is maximal monotone (see (Facchinei and Pang 2003)), allowing for applying the proximal point framework. Hence,

$$
z_{k}^{*}=J_{\lambda}^{T}\left(u_{k}\right) \Longleftrightarrow 0 \in\left(F+\frac{1}{\lambda} \mathbf{I}\right)\left(z_{k}^{*}\right)-\frac{1}{\lambda} u_{k}+\mathscr{N}_{X}\left(z_{k}^{*}\right) \Longleftrightarrow z_{k}^{*} \in \operatorname{SOL}\left(X, F+\frac{1}{\lambda} \mathbf{I}-\frac{1}{\lambda} u_{k}\right),
$$

where $\operatorname{SOL}(X, H+u)$ denotes the solution set of variational inequality problem with set $X$ and mapping $F(x)+u$. It can be immediately seen that if $F$ is a monotone map, then $F+\frac{1}{\lambda} \mathbf{I}$ is a $\frac{1}{\lambda}-$ strongly monotone map. In this section, when $F$ is a monotone expectation-valued map, we develop a proximal framework of (2) reliant on solving a sequence of strongly monotone stochastic variational inequality problems, each of which is solved with increasing exactness. Solving (2) is equivalent to resolving $0 \in T(x) \triangleq \mathbb{E}[F(x, \omega)]+\mathscr{N}_{X}(x)$. We employ a stochastic proximal point framework described in Section 3.2, formalized in Algorithm 2. We proceed to show that the scheme admits a convergence rate of $\mathscr{O}(1 / k)$ where at each step of (19), we obtain an increasingly accurate solution of $J_{\lambda}^{T}\left(u_{k}\right)$ by running Algorithm 1 for a finite (but increasing with $k$ number of iterations denoted by $\ell_{k}$.

Algorithm 2 ( Proximal-point Algorithm with Variable Sample-sizes (PPAWSS)).

(2.0) Given $u_{0} \in X, \lambda>0$ and let $\eta \in(0,2), k=0$;

(2.1) Run VS-Ave $\left(F+1 / \lambda I-1 / \lambda u_{k}, y_{0}, 1 / \lambda, L+1 / \lambda, \ell_{k}\right)$;

(2.2) $\quad z_{k}=\bar{y}_{\ell_{k}}$;

(2.3) $\quad u_{k+1}=\eta z_{k}+(1-\eta) u_{k}$;

(2.4) If $k>K$, then stop; else $k=k+1$; return to (1);

(2.5) Return $u_{K}$.

In the following theorem, we state the convergence result for Algorithm 2.

Theorem 3 (Convergence rate of Stochastic Proximal Point Scheme (PPAWSS)) Suppose $\left\{u_{k}\right\}$ is a sequence generated by Algorithm 2, $T$ is defined as $T(x)=\mathbb{E}[F(x, \omega)]+\mathscr{N}_{X}(x)$, and $T_{\lambda}$ denotes the Yosida approximation operator. Suppose Assumptions 1 and 2 hold. Let $\ell_{k}=\left\lfloor 2 \log _{1 / q}\left((1+k)^{\alpha}\right)\right\rfloor$ for any $\alpha>1$ and $e_{k}=\frac{1}{\lambda}\left(J_{\lambda}\left(x_{k}\right)-z_{k}\right)$.

(i) For any $\lambda>0$ and $K>0$, the following holds:

$$
\mathbb{E}\left[\left\|T_{\lambda}\left(u_{K}\right)\right\|^{2}\right] \leq \frac{\tilde{C}}{\eta(2-\eta) \lambda^{2}(K+1)}+\frac{2 C \eta \hat{C}}{(2-\eta) \lambda(K+1)}=\mathscr{O}(1 /(K+1)),
$$

where $C$ is defined in Theorem $2, \hat{C} \triangleq 2+\frac{1}{2 \alpha-1}+\frac{1}{2(2 \alpha-1)(\alpha-1)}$, and

$$
\tilde{C} \triangleq\left\|u_{0}-u^{*}\right\|^{2}+6 C \eta^{2} \lambda\left(1+\frac{1}{2 \alpha-1}\right)+2 \eta\left\|u_{0}-u^{*}\right\| \sqrt{2 C \lambda}\left(1+\frac{1}{\alpha-1}\right)+4 C \eta^{2} \lambda\left(1+\frac{1}{\alpha-1}\right)^{2} .
$$


(ii) Suppose an $\varepsilon$-solution $u_{k}$ is defined as $\mathbb{E}\left[\left\|T\left(u_{k}\right)\right\|^{2}\right] \leq \varepsilon$. Then computing an $\varepsilon$-solution requires solving $\mathscr{O}\left(\frac{1}{\varepsilon} \log \left(\frac{1}{\varepsilon}\right)\right)$ proximal problems and $\mathscr{O}(1 / \varepsilon)^{1+2 \alpha \beta}$ evaluations of the map.

Proof. (i) Let $\tilde{u}_{k+1} \triangleq u_{k}-\eta \lambda T_{\lambda}\left(u_{k}\right)$. By invoking the definition of $J_{\lambda}^{T}$, we have that:

$$
\begin{aligned}
\left\langle T_{\lambda}\left(u_{1}\right)-T_{\lambda}\left(u_{2}\right), u_{1}-u_{2}\right\rangle & =\left\langle T_{\lambda}\left(u_{1}\right)-T_{\lambda}\left(u_{2}\right), \lambda T_{\lambda}\left(u_{1}\right)-\lambda T_{\lambda}\left(u_{2}\right)\right\rangle+\left\langle T_{\lambda}\left(u_{1}\right)-T_{\lambda}\left(u_{2}\right), J_{\lambda}^{T}\left(u_{1}\right)-J_{\lambda}^{T}\left(u_{2}\right)\right\rangle \\
& =\lambda\left\|T_{\lambda}\left(u_{1}\right)-T_{\lambda}\left(u_{2}\right)\right\|^{2}+\left\langle T_{\lambda}\left(u_{1}\right)-T_{\lambda}\left(u_{2}\right), J_{\lambda}^{T}\left(u_{1}\right)-J_{\lambda}^{T}\left(u_{2}\right)\right\rangle .
\end{aligned}
$$

Using the definition of $\tilde{u}_{k+1},(20)$ and by recalling that $T_{\lambda}\left(u^{*}\right)=0$, we get

$$
\begin{aligned}
\left\|\tilde{u}_{k+1}-u^{*}\right\|^{2} & =\left\|u_{k}-u^{*}-\eta \lambda T_{\lambda}\left(u_{k}\right)\right\|^{2}=\left\|u_{k}-u^{*}\right\|^{2}+\eta^{2} \lambda^{2}\left\|T_{\lambda}\left(u_{k}\right)\right\|^{2}-2 \eta \lambda\left\langle T_{\lambda}\left(u_{k}\right), u_{k}-u^{*}\right\rangle \\
& =\left\|u_{k}-u^{*}\right\|^{2}-\eta(2-\eta) \lambda^{2}\left\|T_{\lambda}\left(u_{k}\right)\right\|^{2}-2 \eta \lambda \underbrace{\left\langle T_{\lambda}\left(u_{k}\right), J_{\lambda}\left(u_{k}\right)-J_{\lambda}\left(u^{*}\right)\right\rangle}_{\geq 0, T_{\lambda}\left(u_{k}\right) \in T\left(J_{\lambda}\left(u_{k}\right)\right), T \text { is monotone }} \\
& \leq\left\|u_{k}-u^{*}\right\|^{2}-\eta(2-\eta) \lambda^{2}\left\|T_{\lambda}\left(u_{k}\right)\right\|^{2} \\
\Longrightarrow & \left\|u_{k+1}-u^{*}\right\| \leq\left\|\tilde{u}_{k+1}-u^{*}\right\|+\overbrace{\left\|u_{k+1}-\tilde{u}_{k+1}\right\|}^{\leq\left\|\eta e_{k}\right\|} \leq\left\|u_{0}-u^{*}\right\|+\eta \lambda \sum_{i=0}^{k}\left\|e_{i}\right\| .
\end{aligned}
$$

Since $\left\|\tilde{u}_{k+1}-u^{*}\right\| \leq\left\|u_{0}-u^{*}\right\|+\eta \lambda \sum_{i=0}^{k}\left\|e_{i}\right\|$, we have the following.

$$
\begin{aligned}
&\left\|u_{k+1}-u^{*}\right\|^{2} \leq\left\|\tilde{u}_{k+1}-u^{*}\right\|^{2}+\left\|u_{k+1}-\tilde{u}_{k+1}\right\|^{2}+2\left\|\tilde{u}_{k+1}-u^{*}\right\|\left\|u_{k+1}-\tilde{u}_{k+1}\right\| \\
& \stackrel{\text { (21),(22) }}{\leq}\left\|u_{k}-u^{*}\right\|^{2}-\eta(2-\eta) \lambda^{2}\left\|T_{\lambda}\left(u_{k}\right)\right\|^{2}+\eta^{2} \lambda^{2}\left\|e_{k}\right\|^{2}+2 \eta \lambda\left\|e_{k}\right\|\left(\left\|u_{0}-u^{*}\right\|+\eta \lambda \sum_{i=0}^{k}\left\|e_{i}\right\|\right) .
\end{aligned}
$$

Summing (23) from $k=0$ to $K$ and taking expectations, we have the following.

$$
\begin{aligned}
& \eta(2-\eta) \lambda^{2} \sum_{k=0}^{K}\left[\left\|T_{\lambda}\left(u_{k}\right)\right\|\right] \\
& \leq\left\|u_{0}-u^{*}\right\|^{2}-\left\|u_{K+1}-u^{*}\right\|^{2}+\sum_{k=0}^{K}\left(\eta^{2} \lambda^{2}\left\|e_{k}\right\|^{2}+2 \eta \lambda\left\|e_{k}\right\|\left(\left\|u_{0}-u^{*}\right\|+\eta \lambda \sum_{i=0}^{k}\left\|e_{i}\right\|\right)\right) \\
& \eta(2-\eta) \lambda^{2} \sum_{k=0}^{K} \mathbb{E}\left[\left\|T_{\lambda}\left(u_{k}\right)\right\|\right] \\
& \leq\left\|u_{0}-u^{*}\right\|^{2}+\eta^{2} \lambda^{2}\left(\sum_{k=0}^{K} \mathbb{E}\left[\left\|e_{k}\right\|^{2}\right]\right)+2 \eta \lambda\left\|u_{0}-u^{*}\right\|\left(\sum_{k=0}^{K} \mathbb{E}\left[\left\|e_{k}\right\|\right]\right)+2 \eta^{2} \lambda^{2} \mathbb{E}\left[\left(\sum_{i=0}^{K} \sum_{j=0}^{i}\left\|e_{j}\right\|\|\| e_{i} \|\right)\right] .
\end{aligned}
$$

If $\hat{\mathscr{F}}_{j} \triangleq\left\{u_{0}, \ldots, u_{j}\right\}$, then from the law of total expectation, we know that for all $j<i, \mathbb{E}\left[\left\|e_{i}\right\|\left\|e_{j}\right\|\right]=$ $\mathbb{E}\left[\mathbb{E}\left[\left\|e_{i}\right\|\left\|e_{j}\right\| \mid \hat{\mathscr{F}}_{j}\right]\right]=\mathbb{E}\left[\left\|e_{j}\right\| \mathbb{E}\left[\left\|e_{i}\right\| \mid \hat{\mathscr{F}}_{j}\right]\right]$. Hence the following holds:

$$
\begin{aligned}
& \eta(2-\eta) \lambda^{2} \sum_{k=0}^{K} \mathbb{E}\left[\left\|T_{\lambda}\left(u_{k}\right)\right\|\right] \leq\left\|u_{0}-u^{*}\right\|^{2}+\eta^{2} \lambda^{2}\left(\sum_{k=0}^{K} \mathbb{E}\left[\left\|e_{k}\right\|^{2}\right]\right)+2 \eta \lambda\left\|u_{0}-u^{*}\right\|\left(\sum_{k=0}^{K} \mathbb{E}\left[\left\|e_{k}\right\|\right]\right) \\
& +2 \eta^{2} \lambda^{2} \mathbb{E}\left[\sum_{k=0}^{K}\left\|e_{k}\right\|^{2}\right]+2 \eta^{2} \lambda^{2} \sum_{i=0}^{K} \sum_{j=0}^{i-1} \mathbb{E}\left[\left\|e_{j}\right\| \mathbb{E}\left[\left\|e_{i}\right\| \mid \hat{\mathscr{F}}_{j}\right]\right] \\
& =\left\|u_{0}-u^{*}\right\|^{2}+3 \eta^{2} \lambda^{2}\left(\sum_{k=0}^{K} \mathbb{E}\left[\left\|e_{k}\right\|^{2}\right]\right)+2 \eta \lambda\left\|u_{0}-u^{*}\right\|\left(\sum_{k=0}^{K} \mathbb{E}\left[\left\|e_{k}\right\|\right]\right)+2 \eta^{2} \lambda^{2} \sum_{i=0}^{K} \sum_{j=0}^{i-1} \mathbb{E}\left[\left\|e_{j}\right\| \mathbb{E}\left[\left\|e_{i}\right\| \mid \hat{\mathscr{F}}_{j}\right]\right] .
\end{aligned}
$$


From Theorem $2, \mathbb{E}\left[\left\|e_{k}\right\|^{2}\right] \leq \frac{2 C}{\lambda} q^{\ell_{k}} \leq \frac{2 C}{\lambda}(k+1)^{-2 \alpha}$, since $\ell_{k} \triangleq\left\lfloor 2 \log _{1 / q}\left((1+k)^{\alpha}\right)\right\rfloor$ for any $\alpha>1$. Therefore,

$$
\sum_{k=0}^{K} \frac{2 C}{\lambda}(k+1)^{-2 \alpha} \leq \frac{2 C}{\lambda}\left(1+\int_{0}^{K}(x+1)^{-2 \alpha} d x\right)=\frac{2 C}{\lambda}\left(1+\frac{(K+1)^{1-2 \alpha}-1}{1-2 \alpha}\right) \leq \frac{2 C}{\lambda}\left(1+\frac{1}{2 \alpha-1}\right) .
$$

Now by substituting (25) in (24) and by Jensen's inequality, we obtain the following.

$$
\begin{aligned}
\eta(2-\eta) \lambda^{2} \sum_{k=0}^{K} \mathbb{E}\left[\left\|T_{\lambda}\left(u_{k}\right)\right\|\right] & \leq\left\|u_{0}-u^{*}\right\|^{2}+6 C \eta^{2} \lambda\left(1+\frac{1}{2 \alpha-1}\right)+2 \eta\left\|u_{0}-u^{*}\right\| \sqrt{2 C \lambda}\left(1+\frac{1}{2 \alpha-1}\right) \\
& +4 C \eta^{2} \lambda\left(1+\frac{1}{2 \alpha-1}\right)^{2} \triangleq \tilde{C} \Longrightarrow \eta(2-\eta) \lambda^{2} \sum_{k=0}^{K} \mathbb{E}\left[\left\|T_{\lambda}\left(u_{k}\right)\right\|\right] \leq \tilde{C} .
\end{aligned}
$$

Since $\left\|T_{\lambda}\left(u_{k+1}\right)\right\|=\left\|T_{\lambda}\left(u_{k+1}\right)-T_{\lambda}\left(u_{k}\right)+T_{\lambda}\left(u_{k}\right)\right\|$, the following holds:

$$
\begin{aligned}
\left\|T_{\lambda}\left(u_{k+1}\right)\right\|^{2} & =\left\|T_{\lambda}\left(u_{k}\right)\right\|^{2}+\left\|T_{\lambda}\left(u_{k+1}\right)-T_{\lambda}\left(u_{k}\right)\right\|^{2}+2\left(T_{\lambda}\left(u_{k+1}\right)-T_{\lambda}\left(u_{k}\right)\right)^{T} T_{\lambda}\left(u_{k}\right) \\
& \leq\left\|T_{\lambda}\left(u_{k}\right)\right\|^{2}-\frac{2-\eta}{\eta}\left\|T_{\lambda}\left(u_{k+1}\right)-T_{\lambda}\left(u_{k}\right)\right\|^{2}+\frac{2}{\eta}\left\|T_{\lambda}\left(u_{k+1}\right)-T_{\lambda}\left(u_{k}\right)\right\|\left\|e_{k}\right\| \\
& \leq\left\|T_{\lambda}\left(u_{k}\right)\right\|^{2}-\frac{2-\eta}{\eta}\left\|T_{\lambda}\left(u_{k+1}\right)-T_{\lambda}\left(u_{k}\right)\right\|^{2}+\frac{2-\eta}{\eta}\left\|T_{\lambda}\left(u_{k+1}\right)-T_{\lambda}\left(u_{k}\right)\right\|^{2}+\frac{\eta}{2-\eta}\left\|e_{k}\right\|^{2} \\
& \leq\left\|T_{\lambda}\left(u_{k}\right)\right\|^{2}+\frac{\eta}{2-\eta}\left\|e_{k}\right\|^{2},
\end{aligned}
$$

where the first inequality arises from adding and subtracting $\frac{2}{\eta}\left\|T_{\lambda}\left(u_{k+1}\right)-T_{\lambda}\left(u_{k}\right)\right\|^{2}$ and the CauchySchwarz inequality while the second inequality follows from Young's inequality. Summing from $k=n$ to $K, \mathbb{E}\left[\left\|T_{\lambda}\left(u_{K}\right)\right\|^{2}\right] \leq \mathbb{E}\left[\left\|T_{\lambda}\left(u_{n}\right)\right\|^{2}\right]+\frac{\eta}{2-\eta} \sum_{k=n}^{K} \mathbb{E}\left[\left\|e_{k}\right\|^{2}\right]$. Using inequality (26) we obtain the following

$$
\begin{array}{r}
\tilde{C} \geq \eta(2-\eta) \lambda^{2} \sum_{k=0}^{K} \mathbb{E}\left[\left\|T_{\lambda}\left(u_{k}\right)\right\|\right] \geq \eta(2-\eta) \lambda^{2}\left((K+1) \mathbb{E}\left[\left\|T_{\lambda}\left(u_{K}\right)\right\|^{2}\right]-\frac{\eta}{(2-\eta)} \sum_{k=0}^{K} \sum_{j=k}^{K-1} \mathbb{E}\left[\left\|e_{j}\right\|^{2}\right] .\right), \\
\Longrightarrow \mathbb{E}\left[\left\|T_{\lambda}\left(u_{K}\right)\right\|^{2}\right] \leq \frac{\tilde{C}}{\eta(2-\eta) \lambda^{2}(K+1)}+\frac{\eta}{(2-\eta)(K+1)} \sum_{k=0}^{K} \sum_{j=k}^{K-1} \mathbb{E}\left[\left\|e_{j}\right\|^{2}\right] .
\end{array}
$$

Using the fact that $\mathbb{E}\left[\left\|e_{k}\right\|^{2} \leq \frac{2 C}{\lambda}(k+1)^{-2 \alpha}\right.$, the following can be obtained:

$$
\mathbb{E}\left[\left\|T_{\lambda}\left(u_{K}\right)\right\|^{2}\right] \leq \frac{\tilde{C}}{\eta(2-\eta) \lambda^{2}(K+1)}+\frac{2 C \eta}{(2-\eta) \lambda(K+1)} \sum_{k=0}^{K} \sum_{j=k}^{K-1}(j+1)^{-2 \alpha}
$$

Note that for any $\alpha>1$ we have that:

$$
\begin{aligned}
\sum_{k=0}^{K} \sum_{j=k}^{K-1}(j+1)^{-2 \alpha} & \leq \int_{0}^{K+1} \int_{0}^{K+1}(x+1)^{-2 \alpha} d x d y \leq \sum_{k=0}^{K-1}\left((k+1)^{-2 \alpha}+\int_{k}^{K-1}(x+1)^{-2 \alpha} d x\right) \\
& =\sum_{k=1}^{K-1}(k+1)^{-2 \alpha}+\frac{1}{2 \alpha-1}\left((k+1)^{1-2 \alpha}-K^{1-2 \alpha}\right) \\
& \leq 1+\int_{0}^{K-1}(x+1)^{-2 \alpha} d x+\frac{1}{2 \alpha-1}\left(1+\int_{0}^{K-1}(x+1)^{1-2 \alpha} d x\right) \\
& \leq 2+\frac{1}{2 \alpha-1}+\frac{1}{2(2 \alpha-1)(\alpha-1)} \Longrightarrow \mathbb{E}\left[\left\|T_{\lambda}\left(u_{K}\right)\right\|^{2}\right] \leq \mathscr{O}(1 /(K+1)) .
\end{aligned}
$$




\section{Jalilzadeh and Shanbhag}

(ii) We showed that there exists a constant $\bar{C}$ where $\left\|T_{\lambda}\left(u_{K}\right)\right\|^{2} \leq \bar{C} /(K+1)$. Hence the minimum number of steps to obtain $\varepsilon$-solution can be computed as follows for $\rho \triangleq q^{\beta}$ where $\beta>1$ :

$$
\sum_{k=0}^{\bar{C} / \varepsilon} \ell_{k} \leq \sum_{k=0}^{\bar{C} / \varepsilon} 2 \log _{1 / q}(1+k)^{\alpha} \leq \int_{0}^{\bar{C} / \varepsilon+1} \frac{2 \alpha}{\log 1 / q} \log (1+x) d x \leq \frac{2 \alpha}{\log 1 / q}((\bar{C} / \varepsilon+2) \log (\bar{C} / \varepsilon+2)) .
$$

The oracle complexity in terms of the number of evaluations of $G(x, \xi)$ can then be bounded as follows:

$$
\sum_{k=0}^{\bar{C} / \varepsilon+1} \sum_{s=0}^{\ell_{k}}\left\lfloor\rho^{-s}\right\rfloor=\sum_{k=0}^{\bar{C} / \varepsilon+1} \frac{(1 / \rho)^{\ell_{k}+1}-1}{1 / \rho-1} \leq \sum_{k=0}^{\bar{C} / \varepsilon+1} \frac{(1+k)^{2 \alpha \beta}}{1-\rho} \leq \frac{\int_{0}^{\bar{C} / \varepsilon+2}(1+x)^{2 \alpha \beta} d x}{1-\rho}=\frac{(2+\bar{C} / \varepsilon)^{1+2 \alpha \beta}}{(1-\rho)(1+2 \alpha \beta)} .
$$

\section{NUMERICAL RESULTS}

In this section, we compare our scheme with the extragradient scheme presented by Iusem et al. (2017) on the following stochastic bimatrix game problem:

$$
\min _{x \in \Delta_{n}} \max _{y \in \Delta_{m}} \mathscr{L}(x, y) \triangleq \mathbb{E}[\langle A(\xi) x, y\rangle]
$$

where $\Delta_{n}$ is an $n$-dimensional simplex. We may recast this saddle point problem (27) as an SVI to find $z=(x, y) \in \Delta_{n} \times \Delta_{m}$ such that $\left[\begin{array}{l}A(\xi)^{T} y \\ -A(\xi) x\end{array}\right]^{T}(z-\bar{z}) \geq 0$, for all $\bar{z} \in \Delta_{n} \times \Delta_{m}$. Suppose the total simulation budget is $1 e 7, n=20$, and $m=10$. Table 1 shows that for different choices of Lipschitz constants (L), our scheme compares well with the extragradient scheme proposed by Iusem et al. (2017). Note that we computed the optimal solution in (27) by solving the sample-average problem using $1 e 6$ samples via $\mathrm{CVx}$. Steplength and sample sizes have been chosen as suggested by Iusem et al. (2017), $\eta<1 /(\sqrt{6} L)$, and $N_{k}=\left\lceil\theta(k+\mu) \ln (k+\mu)^{1+b}\right\rceil$, where $\theta=1, b=10^{-3}$ and $\mu=2+10^{-3}$. In the (PPAWSS) scheme, we set $\eta=1$ and $N_{k}=\left\lfloor\rho^{-k}\right\rfloor$, where $\rho=q^{-\beta}, q=1-(1 /(\kappa+1))$ and $\beta=1.001$.

Table 1: Comparing (PPAWSS) scheme with extragradient method for SVI.

\begin{tabular}{|c||c|c||c|}
\hline \multicolumn{1}{|c||}{} & & \multicolumn{2}{c|}{$\left|\mathscr{L}\left(x_{K}, y_{K}\right)-\mathscr{L}\left(x^{*}, y^{*}\right)\right|$} \\
\hline Lipschitz constant (L) & $\lambda$ & PPAWSS & Extragradient \\
\hline \hline 7.05 & 3500 & $6.4576 \mathrm{e}-05$ & $1.2697 \mathrm{e}-04$ \\
\hline 70.5 & 1200 & $3.5218 \mathrm{e}-04$ & $7.1742 \mathrm{e}-04$ \\
\hline 705 & 40 & $2.5911 \mathrm{e}-03$ & $6.0048 \mathrm{e}-03$ \\
\hline
\end{tabular}

It can be seen in Table 1 , when $L$ increases, a smaller $\lambda$ tends to perform better since the subproblem in line (2) of (PPAWSS) is $\frac{1}{\lambda}$-strongly monotone with condition number $\lambda(L+1 / \lambda)$; therefore to manage the condition number, we reduce $\lambda$.

\section{CONCLUDING REMARKS}

We developed two variable sample-size methods to resolve strongly monotone and monotone SVIs, respectively. In the former setting, a linear convergence rate and near optimal oracle complexity are obtained with a more modest dependence on the condition number for an averaging-based variance-reduced scheme (VS-Ave). In monotone regimes, we develop amongst the first proximal point algorithms with variable sample-sizes (PPAWSS) where strongly monotone subproblems are solved with increasing inexactness via (VS-Ave), achieving the canonical deterministic rate. Finally, numerics suggest that proposed scheme compares well with its extragradient competitor. 


\section{Jalilzadeh and Shanbhag}

\section{REFERENCES}

Corman, E., and X. Yuan. 2014. "A Generalized Proximal Point Algorithm and its Convergence Rate". SIAM Journal on Optimization 24(4):1614-1638.

Facchinei, F., and J.-S. Pang. 2003. Finite-dimensional variational inequalities and complementarity problems. Vol. II. Springer Series in Operations Research. Springer-Verlag, New York.

Ghadimi, S., and G. Lan. 2013. "Stochastic First-and Zeroth-order Methods for Nonconvex Stochastic Programming". SIAM Journal on Optimization 23(4):2341-2368.

Iusem, A., A. Jofré, R. I. Oliveira, and P. Thompson. 2017. "Extragradient Method with Variance Reduction for Stochastic Variational Inequalities". SIAM Journal on Optimization 27(2):686-724.

Jalilzadeh, A., U. V. Shanbhag, J. H. Blanchet, and P. W. Glynn. 2018. "Smoothed Variable Sample-size Accelerated Proximal Methods for Nonsmooth Stochastic Convex Programs". arXiv preprint arXiv:1803.00718.

Jiang, H., and H. Xu. 2008. "Stochastic Approximation Approaches to the Stochastic Variational Inequality Problem". IEEE Transactions on Automatic Control 53(6):1462-1475.

Jofré, A., and P. Thompson. 2019. "On Variance Reduction for Stochastic Smooth Convex Optimization with Multiplicative Noise". Mathematical Programming 174(1):253-292.

Juditsky, A., A. Nemirovski, and C. Tauvel. 2011. "Solving Variational Inequalities with Stochastic Mirror-prox Algorithm". Stochastic Systems 1(1):17-58.

Koshal, J., A. Nedić, and U. V. Shanbhag. 2013. "Regularized Iterative Stochastic Approximation Methods for Stochastic Variational Inequality Problems". IEEE Transactions on Automatic Control 58(3):594-609.

Nesterov, Y., and L. Scrimali. 2011. "Solving Strongly Monotone Variational and Quasi-variational Inequalities". Discrete \& Continuous Dynamical Systems-A 31(4):1383-1396.

Rockafellar, R. T. 1976. "Monotone Operators and the Proximal Point Algorithm". SIAM Journal on Control and Optimization 14(5):877-898.

Shanbhag, U. V., and J. H. Blanchet. 2015. "Budget-constrained Stochastic Approximation". In Proceedings of the 2015 Winter Simulation Conference, edited by L. Yilmaz, W. K. V. Chan, I. Moon, T. M. K. Roeder, C. Macal, and M. D. Rossetti, 368-379. Piscataway, New Jersey, USA: Institute of Electrical and Electronics Engineers, Inc.

Yousefian, F., A. Nedić, and U. V. Shanbhag. 2013. "A Regularized Smoothing Stochastic Approximation (RSSA) Algorithm for Stochastic Variational Inequality Problems". In Proceedings of the 2013 Winter Simulation Conference, edited by R. Pasupathy, S.-H. Kim, A. Tolk, R. Hill, and M. E. Kuhl, 933-944. Piscataway, New Jersey, USA: Institute of Electrical and Electronics Engineers, Inc.

Yousefian, F., A. Nedić, and U. V. Shanbhag. 2017. "On smoothing, regularization, and averaging in stochastic approximation methods for stochastic variational inequality problems". Mathematical Programming 165(1):391-431.

\section{AUTHOR BIOGRAPHIES}

AFROOZ JALILZADEH is a Ph.D. candidate in the Harold and Inge Marcus Department of Industrial and Manufacturing Engineering at Pennsylvania State University. She received her bachelor degree in Mathematics and Applications from University of Tehran in Iran. Her research interests include stochastic optimization, variational inequality problems, convex optimization, and machine learning. Her email address is azj5286@psu.edu.

UDAY V. SHANBHAG is a professor in the Harold and Inge Marcus Department of Industrial and Manufacturing Engineering at Pennsylvania State University. He holds a Ph.D. in Management science and Engineering from Stanford University (2006). His research interest lies in stochastic and nonlinear optimization, nonsmooth analysis and variational inequality problems. His email address is udaybag@psu.edu. 